\title{
A COMPARISON BETWEEN THE EFFECTS OF OZONE AND ALKALINE PEROXIDE TREATMENTS ON TMP PROPERTIES AND SUBSEQUENT LOW CONSISTENCY REFINING
}

\author{
Xue Feng Chang, ${ }^{a}$ James A. Olson, ${ }^{b}$ and Rodger P. Beatson ${ }^{c, *}$ \\ As part of a program to reduce electrical energy consumption in the \\ refining process, the effects of the ozone and alkaline peroxide \\ treatments on fibre and handsheet properties, prior and subsequent to \\ low consistency (LC) refining, were assessed and compared by applying \\ different levels of ozone and a range of peroxide and alkali charges to a \\ primary stage hemlock thermomechanical pulp (TMP). Both highly \\ alkaline peroxide treatments and ozone treatments decreased the \\ specific energy required for strong mechanical pulp. The improvement in \\ pulp strength through alkaline peroxide treatment mainly resulted from \\ pulp surface changes caused by generation of acid groups. The highly \\ alkaline peroxide treatments significantly increased pulp brightness but \\ did not promote the further fibrillation during the subsequent $L C$ refining. \\ On the other hand, ozone treatments provided tensile strength increases, \\ along with small brightness enhancements for the dark hemlock TMP, \\ and increased the tensile gains obtained through LC refining. The effects \\ of ozone treatments on tensile strength before and after LC refining were \\ the result of pulp surface modifications, fibre swelling, and loss of fibre \\ wall integrity due to non-selective chemical attack. High levels of ozone \\ treatment caused tear strength to decrease during subsequent LC \\ refining.
}

Keywords: Ozone; Alkaline peroxide; Low consistency refining; Energy; TMP

Contact information: a: Department of Wood Science, University of British Columbia, Vancouver, BC, Canada V6T 1Z4; b: Pulp and Paper Centre, University of British Columbia, Vancouver, BC, Canada V6T 1Z4; c: British Columbia Institute of Technology, Burnaby, BC, Canada V5G 3H2; * Corresponding author: Rodger_Beatson@bcit.ca

\section{INTRODUCTION}

The advantages of thermomechanical pulping in producing high yield pulp have been eroded by high electrical energy consumption in the process together with globally increasing energy costs. A strategy for reducing energy consumption per tonne of pulp produced involves replacement of high consistency (HC) mainline refining with more energy efficient low consistency (LC) refining (Chang et al. 2010; Muenster 2005). However, the application of energy efficient LC refining in mills is currently limited by the fibre cutting that occurs at high specific energy (Muenster 2005). To overcome this limitation, research in chemical pretreatments prior to LC refining along with new developments, such as reduced intensity and gap control, have drawn attention (Chang et al. 2010; Luukkonen et al. 2009; Olson et al. 2003). 
In a recent investigation (Chang et al. 2010) we have shown that highly alkaline peroxide pretreatments of second-stage HC refined thermomechanical pulp (TMP) resulted in a gain in pulp tensile strength and a higher resistance to fibre cutting during subsequent LC refining, with resulting potential reductions in specific refining energy requirement. The improvement in pulp strength was assigned to the acid groups generated during the treatments, which resulted in increased surface charge on the fibres and fines, leading to increased bonding (Chang et al. 2010; Engstrand et al. 1991). In a similar manner, oxidation of TMP using ozone leads to tensile strength increases, as reported previously by many researchers (Lindholm 1977a; Soteland and Kringstad 1968; Soteland 1971; Soteland 1973; Soteland and Loras 1974), and this is also likely related to the formation of carboxylic acid groups, as noted by Katz and Scallan (1983).

This prior research, as well as the similarities between ozone and alkaline peroxide treatments, indicate that application of either of these two reagents prior to LC refining might lead to significant electrical energy savings. However, it is unlikely that the two chemicals, with their different oxidizing powers, will react with the pulp components in the same manner. A comparison of the effects of alkaline peroxide and ozone treatments of primary stage TMP on pulp properties, prior and subsequent to LC refining, would reveal their relative potential in decreasing the specific energy required to obtain a desired pulp quality and also lead to a better understanding of the mechanisms of pulp strength increase.

In this paper, we report the effects of these two chemical treatments on the fibre and handsheet properties of a primary HC refined TMP, prior and subsequent to laboratory-scale low consistency refining using a blender (Chang et al. 2010). The TMP was treated with different levels of ozone and a range of peroxide and alkali charges.

\section{EXPERIMENTAL}

\section{Material}

The pulp used for chemical treatments and LC refining was a primary HC-refined thermomechanical pulp (TMP) made from whole log hemlock chips in the Andritz pilot mill, Springfield, Ohio. The specific energy applied was $685 \mathrm{kWh} / \mathrm{t}$, giving a freeness of $494 \mathrm{~mL}$. The pulp was treated with $0.4 \%$ DTPA prior to chemical treatments to minimize hydrogen peroxide decomposition and to stabilize ozone (Pan et al. 1984; Presley and Hill 1996). The primary pulp was not screened.

\section{Sample Preparation}

The primary HC refined pulp was acidified to $\mathrm{pH} 2.5$ by mixing the pulp with sulphuric acid in a Hobart Mixer. The acidified pulp was dewatered to approximately $40 \%$ consistency and then fluffed using the Hobart Mixer before alkaline peroxide or ozone treatments.

\section{Ozone Treatments}

The fluffed pulp (150 g oven-dry (O.D.) equivalent at 40\% consistency) was treated with three ozone charges $(1 \%, 3 \%$, and $5 \%$ on OD pulp) in the ozonator. The 
choice of ozone charges was based on the work of Lindholm (1977a). The ozonator consisted of a rotating glass vessel to ensure uniform exposure of ozone to the pulp. The ozone-treated pulps were either soaked in $0.1 \mathrm{M}$ sodium hydroxide $(\mathrm{NaOH})$ at $3.6 \%$ consistency for one hour followed by washing with deionized water or were directly washed using deionized water without $\mathrm{NaOH}$ soaking. During washing, the filtrate was recycled to retain fines.

\section{Alkaline Peroxide Treatments}

The acidified and fluffed pulp was washed with deionized water and then dewatered to around $20 \%$ consistency prior to alkaline peroxide treatments. The washed pulp (30 g O.D. equivalent) was put into a plastic bag and mixed with bleaching liquor at $15 \%$ consistency before being incubated in a water bath at $60{ }^{\circ} \mathrm{C}$ for one hour with occasional mixing. Chemical charges were $0.1 \%$ magnesium sulfate $\left(\mathrm{MgSO}_{4}\right), 3 \%$ sodium silicate $\left(\mathrm{Na}_{2} \mathrm{SiO}_{3}\right)$, $4 \%$ hydrogen peroxide $\left(\mathrm{H}_{2} \mathrm{O}_{2}\right)$, and sodium hydroxide $(0.6 \%$, $3.0 \%$ or $6.0 \%$ ) on O.D. pulp based on our previous work (Chang et al. 2010). The treated pulps were washed with deionized water with recycling of the filtrate to retain fines.

\section{LC Refining with a Waring Blender}

The pulps (12 g O.D. equivalent) after ozone or alkaline peroxide treatments were $\mathrm{LC}$ refined at a laboratory scale using a Waring blender with a $1 \mathrm{~L}$ capacity bowl. The pulps were diluted to $500 \mathrm{~mL}$, then blended at $2.4 \%$ consistency with a power input of $1.9 \mathrm{~A}$ at $115 \mathrm{~V}$ for $20 \mathrm{~min}$ with a pause after $10 \mathrm{~min}$ to cool down the samples to approximately room temperature.

\section{Pulp Freeness}

Freeness of the pulps was tested according to PAPTAC standard method C1. Pulps were hot-disintegrated to remove latency prior to freeness measurements and handsheet preparation.

\section{Handsheet Properties}

Handsheets were made according to PAPTAC method C.4. White water was recycled during handsheet making. Bulk, brightness, tensile strength, and tear resistance were determined according to PAPTAC standards D.4, D.12, D.6H, and D.9 respectively.

\section{Acid Group Content}

Amounts of acid groups in pulps were determined using the method of conductometic titration of pulp as described by Beatson (1992).

\section{Statistics}

The data points in the figures are mean values from the measurements. The measurements are duplicated unless specified. Error bars in all graphs refer to 95\% confidence intervals. 


\section{RESULTS AND DISCUSSION}

Chemical treatment of traditional mechanical pulps produced through high consistency refining has been used as a means to increase pulp quality (Lindholm and Kurdin 1999) and in some cases to reduce energy input. Recently low consistency (LC) refining has been increasingly used in the mechanical pulping process as a means of reducing electrical energy consumption (Musselman et al. 1997). By combining the two technologies, greater gains in pulp quality and energy savings might be possible. The effects of combining alkaline peroxide or ozone treatments with LC refining on a hemlock mechanical pulp refined at high consistency at a specific energy of $685 \mathrm{kWh} / \mathrm{t}$ to $494 \mathrm{~mL}$ CSF have been investigated. Ozone was applied at levels of 1\%, 3\%, and 5\% based on the weight of oven-dried (O.D.) pulp. The effects of subsequent sodium hydroxide soaking of the ozone-treated pulps were also determined. Alkaline peroxide treatments were conducted for one hour using 4\% hydrogen peroxide and three different sodium hydroxide charges of $0.6 \%, 3.0 \%$, and $6.0 \%$ based on the weight of O.D. pulp.

Low consistency refining of the chemically treated pulps was performed on a labscale using a Waring blender under conditions which have been previously shown to mimic the application of a specific energy of $230 \mathrm{kWh} / \mathrm{t}$ in a pilot scale low consistency refiner (Chang et al. 2010).

\section{Tensile Strength}

As seen in Fig.1, tensile strength of the primary HC refined TMP increased with increasing alkali charge for the same amount of hydrogen peroxide applied.

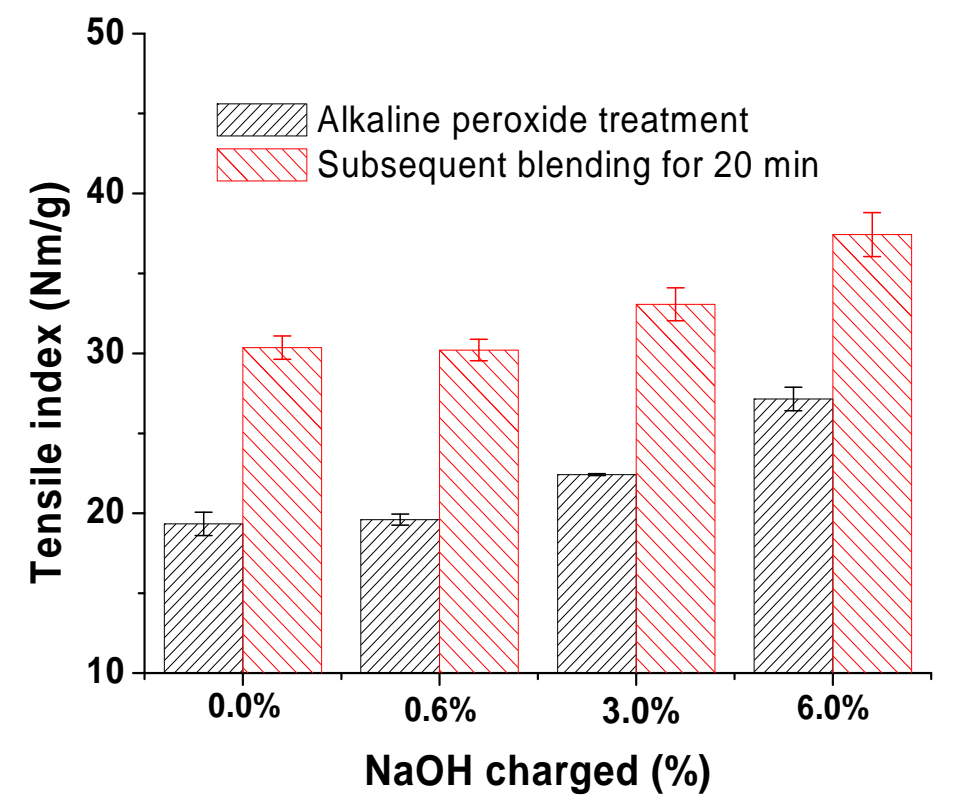

Fig. 1. Treatments with $4 \% \mathrm{H}_{2} \mathrm{O}_{2}$ improved tensile strength at high alkalinity. Tensile gains on LC refining were similar to the control. 
Tensile strength gains on LC refining were the same as the control for all the treatments, indicating that the alkaline peroxide treatments did not have synergistic effects with refining on tensile strength development. Apparently, the alkaline peroxide treatments did not promote further fibrillation of the primary TMP during subsequent LC refining. A similar behaviour was observed previously for a secondary HC refined TMP (Chang et al. 2010).

The ozone treatments improved tensile strength of the primary refined TMP and increased the tensile gain obtained through subsequent LC refining, as indicated by the divergence of the lines for tensile strength before and after blending (Fig. 2a). Soaking of the ozone-treated pulps with $0.1 \mathrm{M}$ sodium hydroxide caused further increases in tensile strength, and again, gains in tensile on LC refining increased with applied ozone charge but only up to a level of $4 \%$ (Fig. 2b). Apparently, in contrast to alkaline peroxide treatment, ozone treatment promotes beatability during subsequent LC refining.

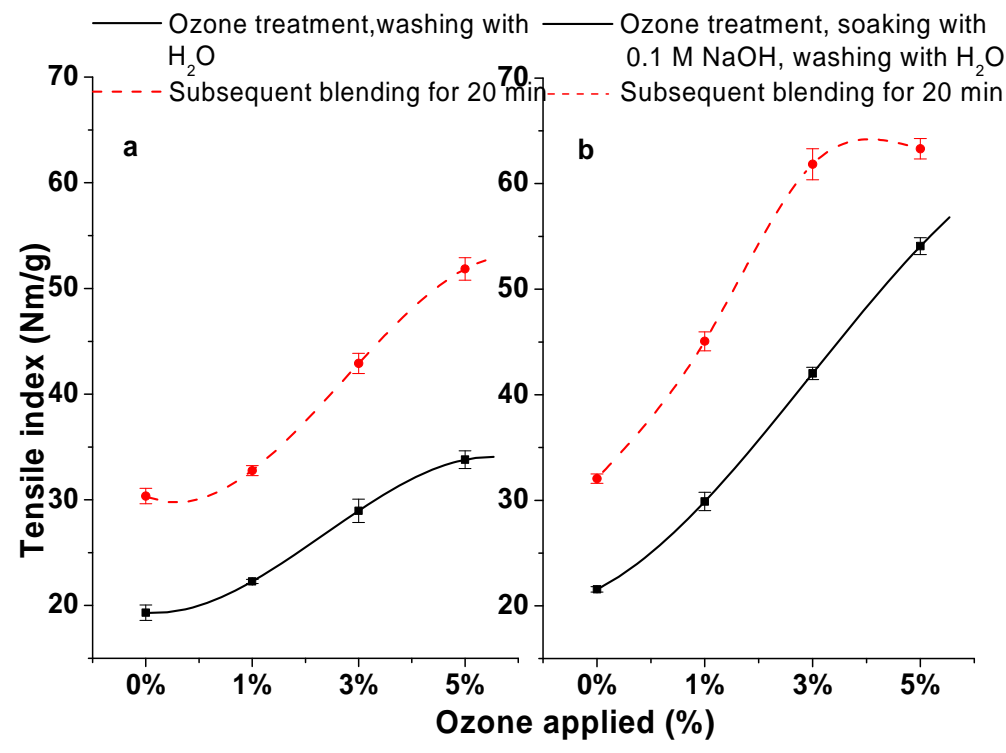

Fig. 2. Ozone treatments improved pulp tensile strength and increased the tensile gain obtained through subsequent LC refining.

The increased tensile strength of the ozone-treated pulps on soaking with sodium hydroxide indicates an important role of pulp swelling in determining the effects of ozone. In contrast to the alkaline peroxide treatments, which were done at high $\mathrm{pH}$ in the presence of sodium ions, the ozone treatments were conducted at $\mathrm{pH} 2.5$ to maintain ozone stability. Thus, the carboxylic acid groups generated during the ozone treatments, as noted in the following section, would be in the hydrogen form and limited fibre wall expansion can be expected due to a low degree of ionization and weak generation of osmotic pressure. Soaking the ozone-treated pulp with sodium hydroxide solution converted the carboxylic acids into the sodium form, completing ionization and maximizing swelling (Scallan 1983).

The enhanced tensile strength and/or the increased response to subsequent LC refining for the primary HC refined TMP achieved by the highly alkaline peroxide and 
ozone treatments indicate that both types of chemical treatment could allow for reduced energy in the second refining stage. For example, a typical fully HC-refined hemlock TMP has a tensile index around $38 \mathrm{Nm} / \mathrm{g}$, requiring approximately 2,000 kWh/t of specific refining energy to produce. The same tensile index can be achieved through treating the primary refined pulp with $4 \%$ hydrogen peroxide and $6 \%$ sodium hydroxide followed by low consistency refining (Fig. 1). The total specific energy application for this process is estimated to be around $1,200 \mathrm{kWh} / \mathrm{t}$ even when the electrical energy required to generate the caustic is included.

\section{Correlation between Tensile Strength and Bulk}

The tensile strength increases of the pulps treated by ozone or alkaline peroxide before and after LC refining were all associated with decreases in handsheet bulk. As seen in Fig. 3, the tensile gains for the ozone-treated pulps were more highly correlated with bulk decrease than those for the alkaline peroxide-treated pulps, indicating that for the former, tensile strength increase is more dependent upon collapse of the fiber wall. For the ozone-treated pulps, the tensile strength gain with bulk drop followed the same trend on LC refining, implying that the gains in tensile on refining mainly arose from a continuation of the same process of increased bonding as a result of further fibre collapse. However, for the alkaline peroxide-treated pulps, on LC refining it was possible to achieve higher tensile at the same bulk. This suggests that, in this instance, the mechanism for tensile development through alkaline peroxide treatments is distinctly different from that of refining. This result is consistent with our previous hypothesis that the tensile strength gain through alkaline peroxide treatments is mainly caused by bond strength increase caused by the surface changes due to the generation of acid groups (Chang et al. 2010). On the other hand, the tensile strength gain through LC refining for these pulps was presumably caused by increased internal fibrillation and fibre compressibility.

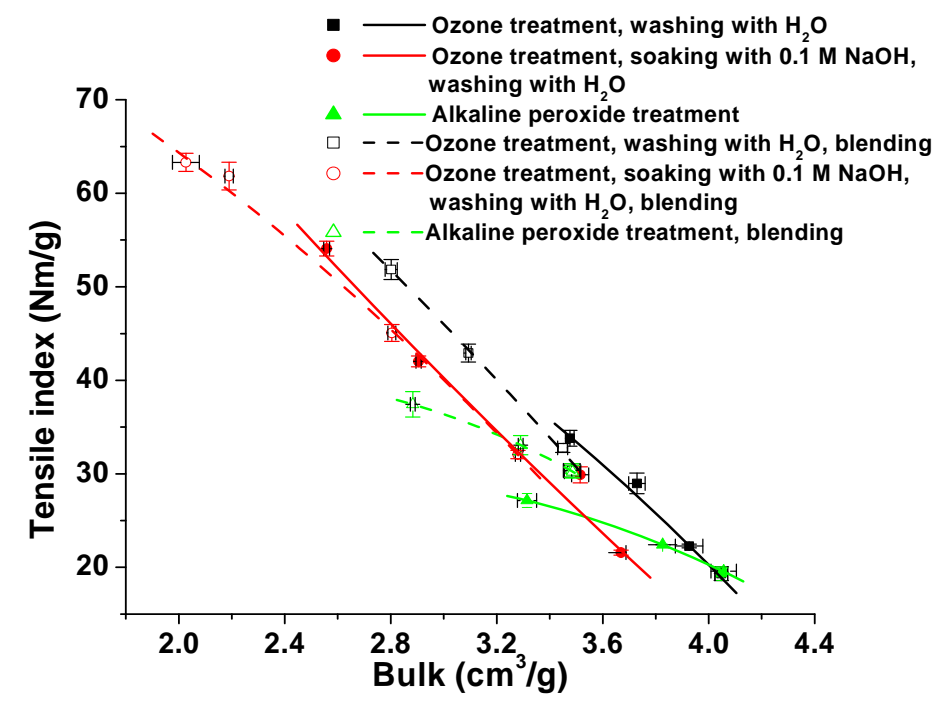

Fig. 3. Tensile strength decreased with handsheet bulk at different rates for the ozone and alkaline peroxide treatments. 
Ozone treatments, as discussed in a later section, not only change the pulp surface but also probably break down cross-links in fibre walls and decrease fibre wall integrity, leading to increased fibre compressibility, which is further enhanced during LC refining. This is consistent with Lindholm's suggestion that ozone treatments cause both fibre surface modification and increase fibre plasticity (Lindholm 1977a).

\section{Correlation between Freeness and Bulk}

Figure 4 shows that there was no significant correlation between the freeness and bulk among the pulps treated by alkaline peroxide, as noted by the constant freeness values. This might indicate that the bulk drops in these handsheets were not due to changes in the compressibility of the fibres in wet state but can be attributed to increased interfibre bonding forces during the drying process, leading to more intimate contact of the surfaces between the fibres or within the lumen. The freeness of the ozone-treated pulp dropped quickly with bulk decrease, which suggests the contribution of highly compressible pulp fibres in the water suspension leading to collapse of the fibre wall during handsheet making. Adding sodium ions to the pulp suspension resulted in a further freeness drop for each sample and a lowering dependence of freeness on bulk. This can be attributed to higher fibre wall swelling and flexibility, plus an increased influence of fibre surface modification. The freeness of each chemically treated pulp dropped significantly with LC refining, presumably through the increased fibrillation and fines generation.

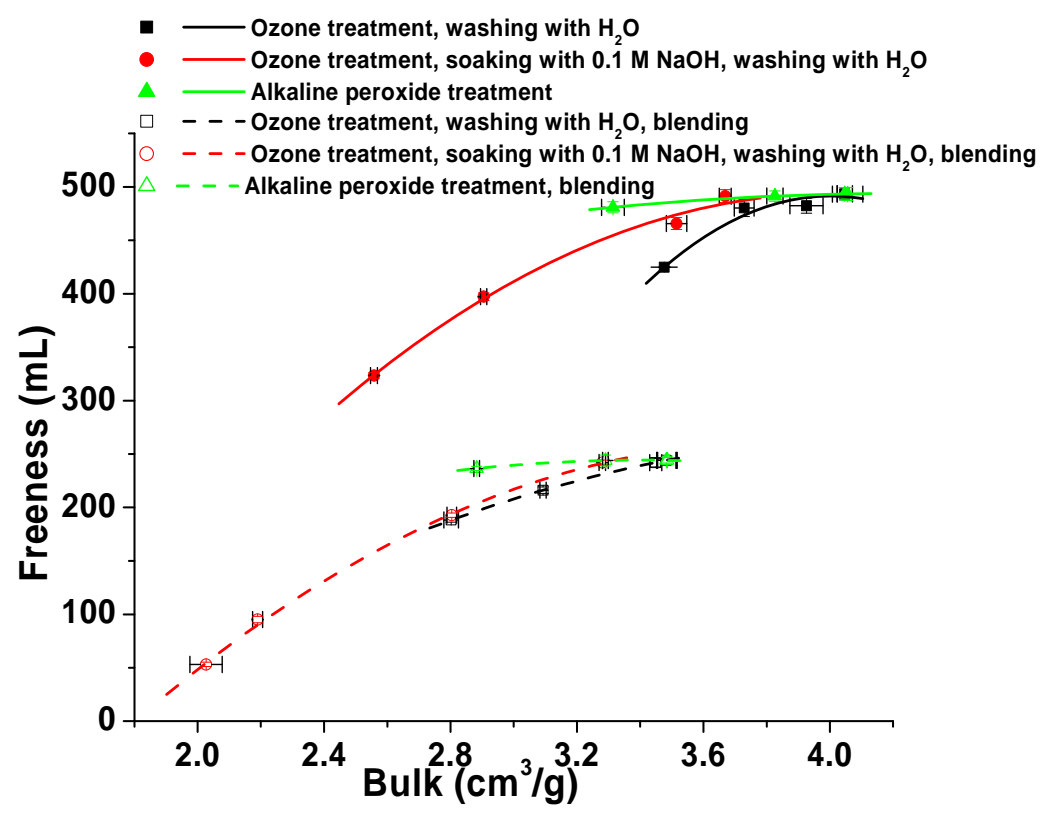

Fig. 4. Freeness drop with bulk occurred on ozone treatment but not on peroxide treatment. LC refining resulted in large freeness decreases at the same bulk for both chemical treatments.

\section{Correlation between Tensile Strength and Acid Group Content}

Figure 5 shows a good correlation between tensile strength and acid group content for both alkaline peroxide and ozone treatments of the primary HC refined TMP. The 
tensile strength increase of the ozone-treated pulps was larger than for the alkaline peroxide-treated pulps at the same level of incorporation of acid groups. Soaking the ozone-treated pulps with sodium hydroxide solution further increased the tensile strength development at a given acid group content.

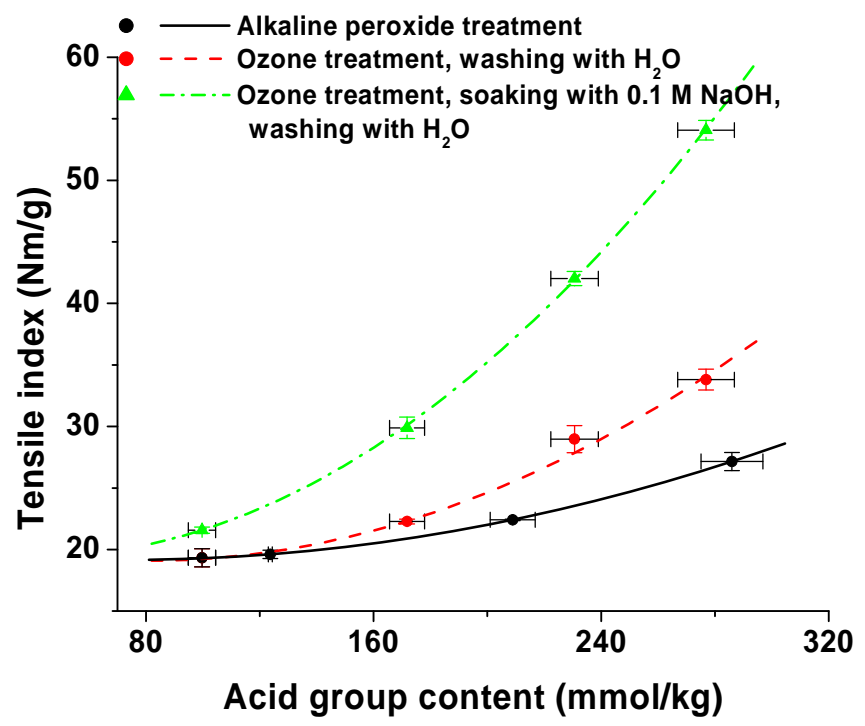

Fig. 5. Tensile strength was correlated with acid group content with different slopes for ozone and alkaline peroxide treatments.

The tensile strength improvement with increased acid group content generated through alkaline peroxide treatments, as discussed previously, was likely the results of bond strength increases arising from surface modification of the fibres or fines. The mechanism of bond strength increase through acid group generation on fibre surfaces is not fully understood (Barzyk et al. 1997). One hypothesis is that surface swelling induced by acid group content can help molecular flexibility, leading to more molecular contact and interdiffusion (Barzyk et al. 1997). In contrast, the higher tensile strength increase with acid group content through ozone treatments probably resulted from a combination of surface modification and interior fibre swelling due to generation of acid groups. The degree of swelling is not only affected by the counter ions but also by the restraining force within the fibre wall (Katz and Scallan 1983). Ozone is highly reactive and nonselective, thus the attack on the fibre wall by ozone could include reaction with lignin, hemicelluloses, and even cellulose, breaking down the molecular crosslinks within fibres and promoting the fibre swelling. The resulting loss of fibre wall integrity, together with fibre swelling, were likely the causes of the synergistic effect on tensile strength seen with subsequent LC refining (Fig. 3).

\section{Correlation between Tensile and Tear Strength}

The tear strength of the pulps treated with either alkaline peroxide or ozone initially increased and then decreased with tensile strength gain, as shown in Fig. 6. This well known phenomenon results from interfibre bonding increases as the tensile strength 
increases. The initial interfiber bonding improvement leads to more work being required to pull the fibres out of the handsheet matrix during the tear test. When interfibre bonding increases to point where fibres are broken rather than being pulled out of the sheet matrix, the tear strength begins to decrease, as less work is then required to break the fibres than to pull them out of sheet matrix.

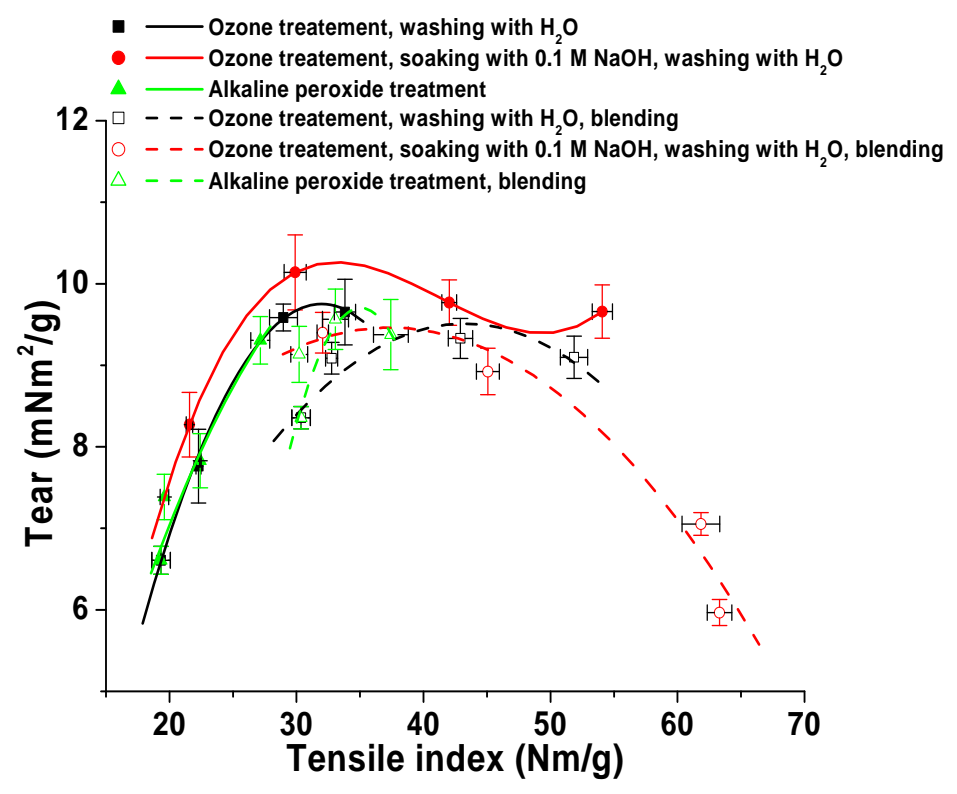

Fig. 6. Tear strength initially increased and then decreased with tensile strength gain for both ozone and alkaline peroxide treatments

The trend of an initial increase followed by a decrease in tear as tensile increases was maintained after the pulps were soaked with sodium hydroxide solution or LC refined. The refining of the alkaline peroxide-treated pulps or ozone-treated pulps, with no added sodium ions, did not lower the tear strength. However, blending of the pulps with ozone treatment followed by soaking with sodium hydroxide solution significantly decreased the tear strength at high tensile levels. This tear decrease might not just be a matter of increased bonding but could indicate that this chemical treatment sufficiently weakened the fibre, resulting in fibre shortening during subsequent LC refining.

\section{Brightness and Opacity}

The ozone treatments had significantly different effects on the brightness and opacity of the primary HC refined pulp when compared to the alkaline peroxide treatments (Table 1). The alkaline peroxide treatments resulted in large increases in the brightness of the pulps along with decreased opacity, both of which were caused by decreasing absorption coefficient as chromophores were removed from the pulps. The maximum brightness gain for the primary HC refined pulps did not occur at the optimal ratio of total alkalinity to peroxide charge for brightness, which is approximately 0.6 for a $4 \% \mathrm{H}_{2} \mathrm{O}_{2}$ application (Presley and Hill 1996). The reason for this was that the primary $\mathrm{HC}$ refined pulp was acidified prior to washing with water and subsequent alkaline 
peroxide treatments, in order to allow a direct comparison of the effects of alkaline peroxide treatments with those obtained through ozone treatments. The ozone treatments slightly increased the brightness of the primary refined pulp. This was different from previous report by Lindholm (1977b) and contrary to our observed brightness drop when ozone was applied to a second-stage HC refined TMP. These different effects of ozone treatments on brightness were probably due to difference in pulp furnishes, as previously been observed by Soteland (1977). The primary refined pulp used in this study was made from whole log hemlock and had a very low initial brightness, while the secondary refined pulp was prepared from a mixture of pine, hemlock, and spruce resulting in a higher initial brightness. Ozone treatments can remove chromophores from the dark hemlock pulp, resulting in a decreased absorption coefficient (Table 1).

Table 1. Ozone and Alkaline Peroxide Treatments Had Different Effects on Brightness and Opacity.

\begin{tabular}{lccc}
\hline Treatment & $\begin{array}{c}\text { Brightness } \\
\text { (ISO) }\end{array}$ & $\begin{array}{c}\text { Opacity } \\
\text { (ISO) }\end{array}$ & $\begin{array}{c}\text { Absorption } \\
\text { coefficient }\left(\mathrm{m}^{2} / \mathrm{kg}\right)\end{array}$ \\
\hline None & 40.04 & 97.18 & 7.18 \\
$1 \% \mathrm{O}_{3}$ & 40.34 & 95.49 & 5.54 \\
$3 \% \mathrm{O}_{3}$ & 40.70 & 95.04 & 4.87 \\
$5 \% \mathrm{O}_{3}$ & 41.61 & 92.92 & 4.41 \\
$\mathrm{NaOH}$ soaking & 38.69 & 95.55 & 8.31 \\
$1 \% \mathrm{O}_{3}+\mathrm{NaOH}$ soaking & 38.92 & 95.92 & 6.72 \\
$3 \% \mathrm{O}_{3}+\mathrm{NaOH}$ soaking & 39.04 & 94.26 & 5.56 \\
$5 \% \mathrm{O}_{3}+\mathrm{NaOH}$ soaking & 37.49 & 94.35 & 6.37 \\
$4 \% \mathrm{H}_{2} \mathrm{O}_{2} \& 0.6 \% \mathrm{NaOH}$ & 52.29 & 92.58 & 3.1 \\
$4 \% \mathrm{H}_{2} \mathrm{O}_{2} \& 3.0 \% \mathrm{NaOH}$ & 63.39 & 87.47 & 1.31 \\
$4 \% \mathrm{H}_{2} \mathrm{O}_{2} \& 6.0 \% \mathrm{NaOH}$ & 64.59 & 86.86 & 1.29 \\
\hline
\end{tabular}

Soaking the ozone-treated pulps with sodium hydroxide solution lowered the brightness by approximately 1.5 ISO due to alkali darkening. The trend to higher brightness for the primary refined pulp with increased dosage of ozone did not change after soaking with sodium hydroxide solution, except for the pulp treated with 5\% ozone. The unexpected drop of brightness for pulp with 5\% ozone treatment on subsequent soaking was probably related to the significant loss of scattering coefficient (Fig. 7) due to the collapse of fibre wall, as discussed above.

\section{Correlation between Tensile Strength and Light Scattering Coefficient}

Figure 7 shows that the tensile strength of the pulps treated with ozone or alkaline peroxide increased with decreased scattering coefficient. This is consistent with the increasing bonded area caused by the two chemical treatments, leading to tensile strength enhancement (Page 1969). At the same scattering coefficient, the alkaline peroxidetreated pulps had higher tensile strengths than the ozone-treated pulps. This could be 
explained by a higher interfibre bond strength for the former, leading to a higher tensile strength at a given bonded area (Page 1969) and is consistent with the observations made in previous sections.

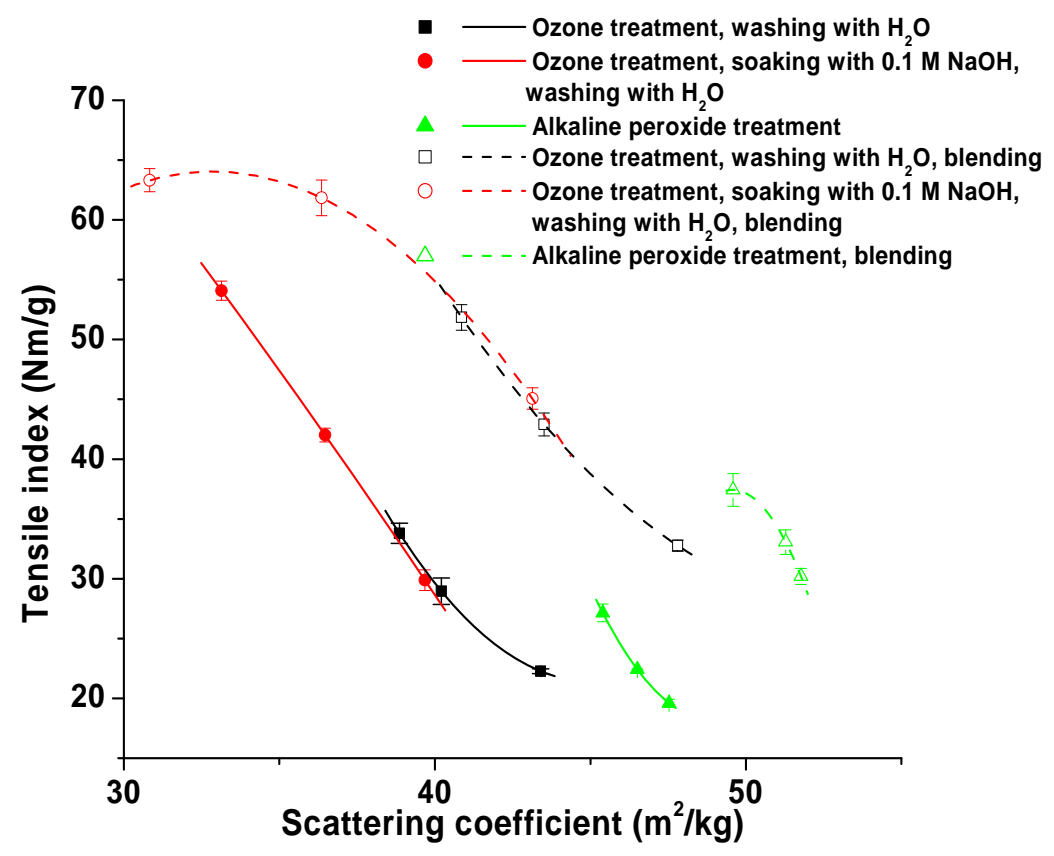

Fig. 7. Tensile strength increased with decreased scattering coefficient for ozone and alkaline peroxide treatments

The subsequent LC refining generally increased both the scattering coefficients and tensile strength of the chemically treated pulps due to increased fibrillation. The scattering coefficient drop, on subsequent LC refining, of the pulp treated with $5 \%$ ozone and soaked in sodium hydroxide likely results from significant fiber wall collapse due to reduced fibre wall rigidity and reduced resistance to beating.

\section{CONCLUSIONS}

1. Both highly alkaline peroxide treatments and ozone treatments can decrease the specific energy required to produce strong mechanical pulp.

2. Highly alkaline peroxide treatments improve the strength of the pulp but do not promote increased fibrillation during subsequent LC refining. The improvement of the pulp strength by alkaline peroxide treatments mainly results from bond strength increase through the generation of acid groups on the surfaces of fibres and fines.

3. Ozone treatments increase tensile strength and promote tensile development during LC refining. The tensile strength increase and additional tensile gains through LC refining caused by the ozone treatments are the result of pulp surface modifications, fibre swelling, and loss of fibre wall integrity due to non-selective chemical attack. The effects of ozone treatments on tensile strength are enhanced by the addition of 
counter sodium ions to the carboxylic acid groups. However, high ozone treatment levels lead to low tear strengths on subsequent LC refining.

4. Ozone and alkaline peroxide treatments have different impacts on the pulp optical properties. For a dark hemlock TMP, alkaline peroxide treatments lead to large increases in brightness along with decreased opacity, while increases in brightness are small for ozone treatments.

\section{ACKNOWLEDGMENTS}

This work was funded by the Natural Sciences and Engineering Research Council of Canada through the Collaborative Research and Development program and through the support of our partners BC Hydro, Paprican, Catalyst Papers, Howe Sound Pulp and Paper, West Fraser Quesnel River Pulp, Canfor, Andritz, Arkema, Honeywell, WestCan Engineering, Advanced Fibre Technologies, Ontario Power Authority and CEATI International. Special thanks go to Marc Sabourin and Eric Xu of Andritz for assistance with pilot trials and to Andritz for use of their pilot facilities.

\section{REFERENCES CITED}

Barzyk, D., Page, D. H., and Ragauskas, A. (1997). "Carboxylic acid groups and fibre bonding," Proc., The Fundamentals of Papermaking Materials: Transactions of the 11th Fundamental Research Symposium, Cambridge, 893-907.

Beatson, R. P. (1992). "Determination of sulfonate groups and total sulfur," Methods in Lignin Chemistry, Springer, Berlin Heidelberg, 473-484.

Chang, X. F., Bridges, C., Vu, D., Kuan, D., Kuang, L., Olson, J. A., Luukonen, A., and Beatson, R. P. (2010). "Saving electrical energy by alkaline peroxide treatment of TMP prior to low consistency refining," Journal of Pulp and Paper Science 36(3-4), 129-134.

Engstrand, P., Sjogren, B., Olander, K., and Htun, M. (1991). "The significance of carboxylic groups for the physical properties of mechanical pulp fibers," Proc., 6th International Symposium Wood Pulping Chemistry, 75-79.

Katz, S., and Scallan, A. M. (1983). "Ozone and caustic soda treatments of mechanical pulp," Tappi J. 66(1), 85-87.

Lindholm, C. A., and Kurdin, J. A. (1999). "Chemimechanical pulping," Mechanical Pulping, Fapet Oy, Helsinki, Finland, 222-249.

Lindholm, C. A. (1977a). "Ozone treatment of mechanical pulp. Part II . Influence on strength properties," Paperi ja Puu - Paper and Timber 59(2), 47-62.

Lindholm, C. A. (1977b). "Ozone treatment of mechanical pulp. Part III. Influence on optical properties," Paperi ja Puu - Paper and Timber 59(NA4), 217-232.

Luukkonen, A., Olson, J. A., and Martinez, D. M. (2009). "Predicting refiner gap and pulp quality in LC refining of mechanical pulp," Proc., International Mechanical Pulping Conference, 195-202. 
Muenster, H. (2005). "Energy savings in TMP by high termperature LC/MC refining," Proc., International Mechanical Pulping Conference, Oslo, Norway.

Musselman, R., Letarte, D., and Simard, R. (1997). "Third stage low consistency refining of TMP for energy savings and quality enhancement," Proc., 4th Int. Refining Conference, Fiuggi, Italy.

Olson, J. A., Drozdiak, J., Martinez, M., Garner, R., Robertson, A. G., and Kerekes, R. (2003). "Characterizing fibre shortening in low-consistency refining using a comminution model," Powder Technol. 129(1-3), 122-129.

Page, D. H. (1969). "A theory for tensile strength of paper," Tappi 52(4), 674-681.

Pan, G., Chen, C. L., Chang, H. M., and Gratzl, J. (1984). "Studies on ozone bleaching. I. The effect of $\mathrm{pH}$, temperature, buffer systems and heavy metal-ions on stability of ozone in aqueous solution," J. Wood Chem. Technol. 4(3), 367-387.

Presley, J. R., and Hill, R. T. (1996). "Peroxide bleaching of (chemi)mechanical pulps," Pulp Bleaching: Principles and Practice, TAPPI, Atlanta, Georgia, 457-489.

Scallan, A. M. (1983). "The effect of acidic groups on the swelling of pulps: A review," Tappi J. 66(11), 73-75.

Soteland, N. (1977). "The effect of ozone on mechanical pulps," Pulp and Paper Canada 78(7), 45-48.

Soteland, N., and Loras, V. (1974). "Effect of ozone on mechanical pulps," Norsk Skogindustri 28(6), 165-169.

Soteland, N. (1973). "Effect of ozone on some properties of thermomechanical pulps," Norsk Skogindustri 27(10), 274-277.

Soteland, N. (1971). "Effect of ozone on some properties of groundwoods of four species. Part I," Norsk Skogindustri 25(3), 61-66.

Soteland, N., and Kringstad, K. (1968). "Effect of ozone on some properties of high-yield pulps," Norsk Skogindistri 22(2), 46-52.

Article submitted: August 19, 2011; Peer review completed: October 16, 2011; Revised version received and accepted: November 2, 2011; Published: November 5, 2011. 